\title{
BMJ Open Retrospective cohort study of new-onset atrial fibrillation in acute pulmonary embolism on prognosis
}

To cite: Liu D, Shi S, Liu X, et al. Retrospective cohort study of new-onset atrial fibrillation in acute pulmonary embolism on prognosis. BMJ Open 2021;11:e047658. doi:10.1136/ bmjopen-2020-047658

- Prepublication history for this paper is available online. To view these files, please visit the journal online (http://dx.do org/10.1136/bmjopen-2020047658).

Received 07 December 2020 Accepted 04 September 2021

Check for updates

(c) Author(s) (or their employer(s)) 2021. Re-use permitted under CC BY-NC. No commercial re-use. See rights and permissions. Published by BMJ.

${ }^{1}$ Department of Cardiology, Renmin Hospital of Wuhan University, Wuhan, People's Republic of China

${ }^{2}$ Cardiovascular Research Institute, Wuhan, People's Republic of China

${ }^{3}$ Hubei Key Laboratory of Cardiology, Wuhan, People's Republic of China

Correspondence to Qingyan Zhao; ruyan71@163.com

\section{ABSTRACT}

Objectives To investigate the characteristics of new-onset atrial fibrillation (AF) and its impact on prognosis in acute pulmonary embolism (aPE).

Design A retrospective cohort study

Setting The study cohort included patients diagnosed with aPE who were admitted to the Renmin Hospital of Wuhan University from January 2017 to January 2019. Participants Patients were $\geq 18$ years of age and hospitalised for aPE.

Outcome measures AF was diagnosed based on an ECG recording or a Holter monitor during hospitalisation. aPE was diagnosed by CT pulmonary angiography. The prescription was determined from the discharge medication list. All-cause mortality was observed after 6-month follow-up. The logistic regression model and Cox proportional hazards model were used to study the risk factor of the new-onset AF and the predictor of all-cause mortality, respectively.

Results A total of 590 patients with aPE were enrolled, 23 $(3.9 \%)$ in the new-onset paroxysmal AF group, 31 (5.3\%) in the new-onset persistent AF group and $536(90.8 \%)$ in the sinus rhythm (SR) group. The incidence of the newonset AF was $9.2 \%(54 / 590)$. A significant difference in age, heart rate, cardiac troponin I ultra, amino-terminal pro-brain natriuretic peptide, D-dimer, left atrial diameter, left ventricular ejection fraction, pulmonary infection, venous thromboembolism, congestive heart failure, chronic cor pulmonale and ischaemic heart disease was found among the three groups $(p<0.05)$. Risk factors for the new-onset AF were massive PE, ischaemic heart disease and congestive heart failure. The survival rate of the paroxysmal and persistent AF group was significantly lower than that of the SR group within 6 months $(60.9 \%$ and $51.6 \%$ vs $88.8 \%, p<0.001)$. New-onset persistent AF (OR 2.73; $95 \% \mathrm{Cl} 1.28$ to $5.81 ; p=0.009$ ) was an independent predictor affecting the 6-month survival in aPE patients.

Conclusions Massive PE, ischaemic heart disease and congestive heart failure are high-risk factors which were related to new-onset AF in aPE. New-onset persistent AF was an independent predictor for 6-month all-cause mortality in PE patients.

\section{BACKGROUND}

The incidence of acute pulmonary embolism (aPE) has gradually increased in the

\section{Strengths and limitations of this study}

- Our study is the largest cohort study on acute pulmonary embolism (aPE) combined with atrial fibrillation (AF) in China, and we first confirmed new-onset persistent $A F$ is an independent predictor for aPE.

- Previous studies did not distinguish AF types, while AF was more carefully divided into paroxysmal and persistent in our study.

- The study was a retrospective analysis of patient medical records and the follow-up time was relatively short.

past decades due to increased old people. ${ }^{1}$ PE causes more than 0.3 million deaths each year in the USA and European countries, ranking third among mortality rates from cardiovascular diseases. ${ }^{2}{ }^{3} \mathrm{PE}$ contributes to the $5 \%-10 \%$ of deaths in hospitalised patients, and venous thromboembolism (VTE) is a leading preventable cause of in-hospital death. ${ }^{45}$ Even though the global data show that the mortality rate from aPE decreases every year, ${ }^{6}$ it may be related to more effective treatments, interventions and strict adherence to guidelines, but thromboembolism is estimated to have caused one in four deaths worldwide in $2010 .^{7}$ aPE has many complications due to haemodynamic disturbances. Cardiovascular disease is the main comorbidity or complication in aPE, which affects the prognosis of patients. Spencer et al demonstrated that congestive heart failure is an independent predictor of mortality in patients with aPE and the short-term mortality rate was $11.1 \%$ $22.2 \%{ }^{8}{ }^{9}$ Besides, a previous study reported that patients with aPE are at higher risk of developing a subsequent cardiovascular disease that affects the prognosis. ${ }^{10}$ However, the pathophysiological mechanism and the effects on prognosis of an episode of atrial fibrillation (AF) as a complication of aPE is still not yet studied enough. Therefore, 
this study aimed to investigate whether the new-onset AF might be considered a marker of the severity of aPE and its impact on the prognosis.

\section{METHOD}

\section{General information}

The study cohort included patients diagnosed with aPE admitted to the Renmin Hospital of Wuhan University from January 2017 to January 2019. The inclusion criteria were the following: (1) patients were all over the age of 18 and their medical records were retrospectively analysed; (2) the diagnosis of aPE was made according to the guidelines for the diagnosis and treatment of aPE developed by the ESC in 2014. ${ }^{11}$ All patients were diagnosed using CT pulmonary angiography. The diagnosis of congestive heart failure is based on echocardiography, biochemistry and clinical symptoms. All stages of congestive heart failure were included. It is necessary that medical history records have coronary angiography, exercise treadmill test or apparent signs of myocardial ischaemia on the ECG during hospitalisation for the diagnosis of ischaemic heart disease. Pulmonary infection is diagnosed by CT or X-ray of chest radiographs.

The exclusion criteria were the following: (1) patients with other arrhythmias, such as supraventricular or ventricular tachycardia; (2) patients with valvular heart disease, previous AF as shown by the medical records, chronic PE or multiple organ failure; (3) patients with acute myocardial infarction and (4) patients who died in hospital (figure 1).
The final number of patients enrolled in this study was 590, and they were divided into three groups, (a) sinus rhythm (SR) group, which included patients with SR at admission; (b) paroxysmal AF group, which included patients with one or more episodes of paroxysmal AF documented by ECG at any time during index hospitalisation; (c) persistent AF group, which included patients suffering from AF episodes that lasted for more than 48 hours, failed to recover spontaneously and required medical or non-pharmacological intervention during the index hospitalisation. All AF cases in this cohort were new-onset (figure 1).

Demographic and clinical characteristics on admission, including symptoms, haemodynamic profile and comorbidities, were evaluated and compared among groups. Besides, the Simplified Pulmonary Embolism Severity Index (sPESI) was retrospectively calculated. ${ }^{12}$ Blood routine (Sysmex HST302, Japan), biochemistry (Siemens ADVIA 2400, Germany), cardiac troponin I ultra (cTnI-ultra, Siemens ADVIA CENTAUR XP, Germany), amino-terminal pro-brain natriuretic peptide (NT-proBNP, Roche, Switzerland) and blood gas analysis (GEM 4000, America) were also measured at admission. Transthoracic echocardiography was performed within 24 hours of admission. Haemodynamic parameters were measured using Philips iE33 (Philips Medical Systems, Netherlands) equipped with S5-1 sensors or GE Vivid 7 (GE Healthcare, America) equipped with M4S sensors. After the patient was diagnosed with aPE, low molecular weight heparin was administered subcutaneously

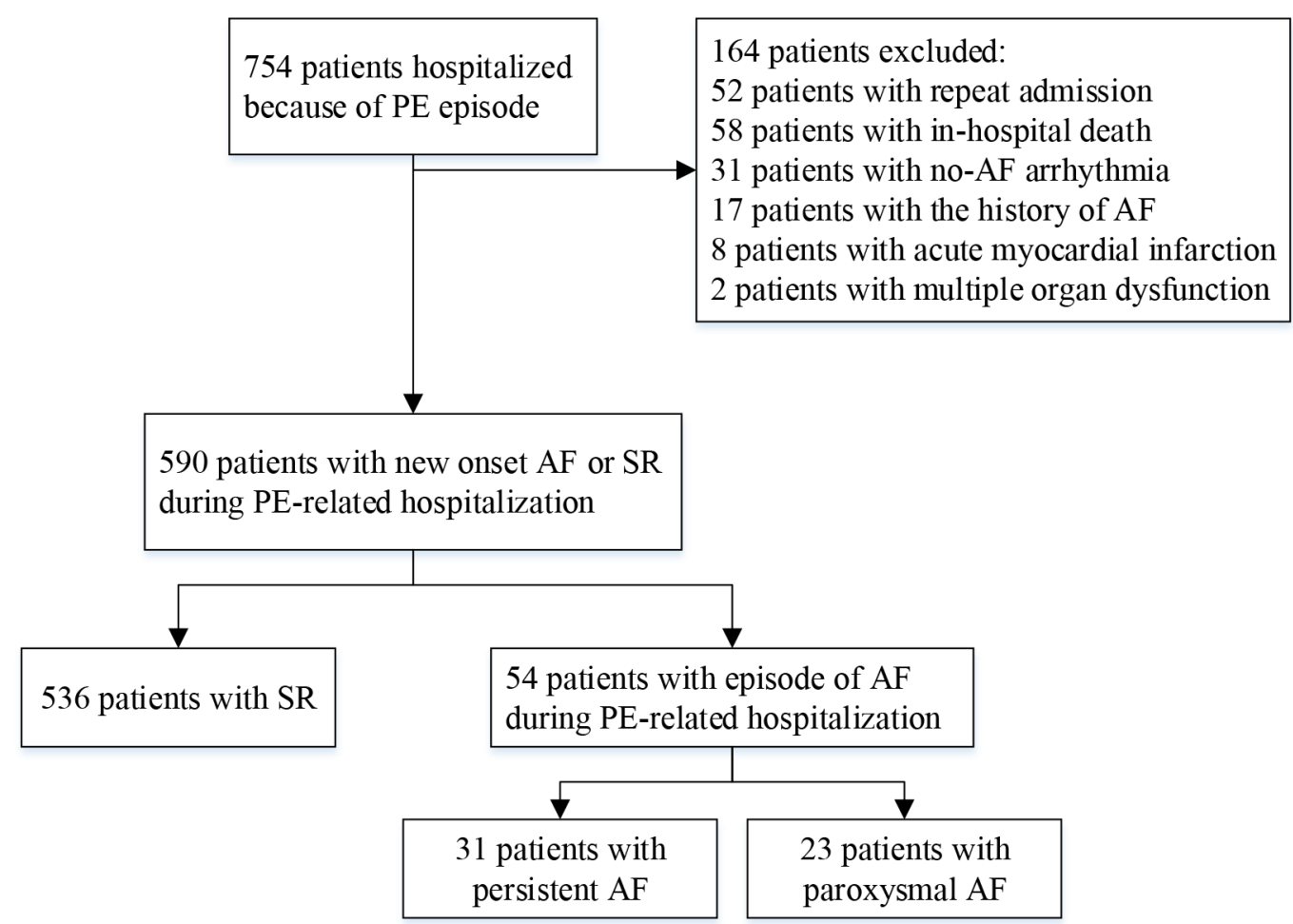

Figure 1 Study population, inclusion and exclusion criteria based on patient's heart rhythm. Study population, and inclusion and exclusion criteria based on patient's heart rhythm. AF, atrial fibrillation; SR, sinus rhythm; PE, pulmonary embolism. 
Table 1 Baseline demographic and laboratory parameters with and without new-onset AF on admission

\section{All patients $(n=590) \quad$ SR $(n=536)$}

Baseline

\begin{tabular}{|c|c|c|c|c|c|}
\hline Age (years) & $67 \pm 14$ & $67 \pm 15$ & $74 \pm 10^{*}$ & $71 \pm 11$ & 0.026 \\
\hline Gender (male) & $287(48.6 \%)$ & $259(48.3 \%)$ & $14(60.9 \%)$ & $14(45.2 \%)$ & 0.46 \\
\hline $\begin{array}{l}\text { Body mass index } \\
\left(\mathrm{kg} / \mathrm{m}^{2}\right)\end{array}$ & $23(19-26)$ & $23(19-26)$ & $22(19-26)$ & $23(20-25)$ & 0.78 \\
\hline $\begin{array}{l}\text { Systolic blood } \\
\text { pressure }(\mathrm{mm} \mathrm{Hg})\end{array}$ & $130 \pm 22$ & $130 \pm 22$ & $122 \pm 20$ & $127 \pm 25$ & 0.13 \\
\hline $\begin{array}{l}\text { Diastolic blood } \\
\text { pressure }(\mathrm{mm} \mathrm{Hg})\end{array}$ & $75 \pm 14$ & $75 \pm 14$ & $74 \pm 14$ & $74 \pm 12$ & 0.96 \\
\hline Heart rate (bpm) & $85 \pm 16$ & $85 \pm 14$ & $88 \pm 24$ & $96 \pm 30^{\star \star \star}$ & 0.001 \\
\hline $\mathrm{pH}$ & $7.42(7.35-7.49)$ & $7.42(7.35-7.49)$ & $7.42(7.41-7.44)$ & $7.43(7.34-7.45)$ & 0.83 \\
\hline $\mathrm{PO}_{2}(\mathrm{~mm} \mathrm{Hg})$ & 75 (64-85) & $75(64-84)$ & $80(64-93)$ & $64(61-88)$ & 0.06 \\
\hline $\mathrm{O}_{2}$ sat $(\%)$ & 92 (88-95) & 92 (88-95) & $94(90-97)$ & 92 (90-95) & 0.38 \\
\hline $\mathrm{PCO}_{2}(\mathrm{~mm} \mathrm{Hg})$ & $38 \pm 10$ & $37 \pm 10$ & $39 \pm 11$ & $38 \pm 10$ & 0.75 \\
\hline Haemoglobin (g/L) & $121(103-138)$ & $121(103-137)$ & $121(88-141)$ & $124(104-144)$ & 0.82 \\
\hline Creatinine (umol/L) & $273 \pm 133$ & $271 \pm 130$ & $328 \pm 167^{*}$ & $266 \pm 153$ & 0.12 \\
\hline NT-proBNP (pg/mL) & 1564 (794-3309) & 1447 (780-3222) & 2356 (1084-4097) & $3121(1213-5260)^{\star \star}$ & 0.002 \\
\hline cTNI-ultra (ng/mL) & $0.057(0.029-0.075)$ & $0.063(0.031-0.075)$ & $0.035(0.015-0.103)$ & $0.016(0.008-0.107)^{*}$ & 0.04 \\
\hline D-dimers (mg/L) & $4.00(2.79-6.46)$ & $4.04(2.86-6.50)$ & $4.31(2.14-9.21)$ & $3.09(1.63-5.16)^{\star \star}$ & 0.009 \\
\hline \multicolumn{6}{|l|}{ Echocardiography } \\
\hline MPAD (mm) & $31 \pm 8$ & $31 \pm 8$ & $32 \pm 7$ & $28 \pm 7$ & 0.17 \\
\hline $\mathrm{LAD}(\mathrm{mm})$ & $38 \pm 9$ & $38 \pm 9$ & $42 \pm 9$ & $42 \pm 7^{*}$ & 0.011 \\
\hline LVDD (mm) & $47 \pm 9$ & $47 \pm 9$ & $49 \pm 7$ & $51 \pm 6^{\star}$ & 0.054 \\
\hline Chest pain & 57 (9.7\%) & $48(9.0 \%)$ & $3(13.0 \%)$ & $6(19.4 \%)$ & 0.14 \\
\hline Chest tightness & $102(17.3 \%)$ & 87 (16.2\%) & 7 (30.4\%) & $8(25.8 \%)$ & 0.09 \\
\hline Syncope & $49(8.3 \%)$ & $44(8.2 \%)$ & $2(8.7 \%)$ & $3(9.7 \%)$ & 0.96 \\
\hline Cough & 273 (46.3\%) & $253(47.2 \%)$ & $10(43.5 \%)$ & 10 (32.3\%) & 0.26 \\
\hline Haemoptysis & $78(13.2 \%)$ & $69(12.9 \%)$ & $4(17.4 \%)$ & $5(16.1 \%)$ & 0.73 \\
\hline \multicolumn{6}{|l|}{ Comorbidity } \\
\hline Malignancy & $66(11.2 \%)$ & $60(11.2 \%)$ & $1(4.3 \%)$ & $5(16.1 \%)$ & 0.40 \\
\hline Septicaemia & 55 (9.3\%) & $47(8.8 \%)$ & $4(17.4 \%)$ & $4(12.9 \%)$ & 0.30 \\
\hline COPD & $47(8.0 \%)$ & $40(7.5 \%)$ & $3(13.0 \%)$ & $4(12.9 \%)$ & 0.36 \\
\hline $\begin{array}{l}\text { Pulmonary artery } \\
\text { hypertension }\end{array}$ & 56 (9.5\%) & $51(9.5 \%)$ & 0 & $5(16.1 \%)$ & 0.14 \\
\hline History of VTE & $180(30.5 \%)$ & 175 (32.6\%) & $3(13.0 \%)$ & $2(6.5 \%)^{\star \star}$ & 0.005 \\
\hline Cerebral infarction & $79(13.4 \%)$ & 67 (12.5\%) & $5(21.7 \%)$ & 7 (22.6\%) & 0.14 \\
\hline Diabetes & $28(4.7 \%)$ & $23(4.3 \%)$ & $3(13.0 \%)$ & $12(6.5 \%)$ & 0.14 \\
\hline $\begin{array}{l}\text { Ischaemic heart } \\
\text { disease }\end{array}$ & 76 (12.9\%) & $59(11.0 \%)$ & $9(39.1 \%)^{\star \star}$ & $8(25.8 \%)^{*}$ & $<0.001$ \\
\hline
\end{tabular}

Continued 
Table 1 Continued

\begin{tabular}{|c|c|c|c|c|c|}
\hline & All patients $(n=590)$ & SR $(n=536)$ & Paroxysmal AF $(n=23)$ & Persistent AF $(n=31)$ & P value \\
\hline $\begin{array}{l}\text { Chronic cor } \\
\text { pulmonale }\end{array}$ & $25(4.2 \%)$ & $20(3.7 \%)$ & $1(4.3 \%)$ & $4(12.9 \%)^{*}$ & 0.048 \\
\hline $\begin{array}{l}\text { Congestive heart } \\
\text { failure }\end{array}$ & $124(21.0 \%)$ & $98(18.3 \%)$ & $10(43.5 \%)^{\star \star}$ & $16(51.6 \%)^{\star \star \star}$ & $<0.001$ \\
\hline \multicolumn{6}{|l|}{ Risk stratification } \\
\hline Massive PE & $125(21.2 \%)$ & $89(16.6 \%)$ & $10(43.5 \%)$ & $13(41.9 \%)$ & \\
\hline Submassive PE & $332(56.3 \%)$ & $312(58.2 \%)$ & $9(39.1 \%)$ & $11(35.5 \%)$ & \\
\hline Non-massive PE & $133(22.5 \%)$ & $135(25.2 \%)$ & $4(17.4 \%)$ & 7 (22.6\%) & $<0.001$ \\
\hline
\end{tabular}

${ }^{*},{ }^{* *},{ }^{* * *}$ : AF group vs SR group, $\mathrm{p}<0.05, \mathrm{p}<0.01$ and $\mathrm{p}<0.001$, respectively.

AF, atrial fibrillation; COPD, chronic obstructive pulmonary disease; cTnl-ultra, cardiac troponin I; LAD, left atrium diameter; LVDD, left ventricular end-diastolic dimension; LVEF, left ventricular ejection fraction; MPAD, main pulmonary artery diameter; NT-proBNP, aminoterminal pro-brain natriuretic peptide; $\mathrm{O}_{2}$ sat, peripheral oxygen saturation; $\mathrm{PCO}_{2}$, partial pressure of carbon dioxide; PE, pulmonary embolism; $\mathrm{PO}_{2}$, blood oxygen pressure; RAD, right atrium diameter; RVDD, right ventricular end-diastolic dimension; RV/LV, right ventricle/left ventricle; sPESI, Simplified Pulmonary Embolism Severity Index; SR, sinus rhythm; VTE, venous thromboembolism.

at a dose of $100 \mathrm{IU} / \mathrm{kg}$ every 12 hours without contraindications. As regard patients using warfarin, the international normalised ratio (INR) target is usually set at 2.0-3.0. Regarding patients using novel oral anticoagulants (NOAC), dabigatran $110 \mathrm{mg}$ twice daily or rivaroxaban $20 \mathrm{mg}$ once daily recommended.

\section{Follow-up}

All-cause mortality was measured by telephone, message and email at 6 month after discharge.

\section{Statistical analysis}

Statistical analysis was performed using SPSS Statistics V.25.0 software (IBM Corp, New York, USA). Patients' characteristics were presented as frequencies for the categorical variables and as mean $\pm \mathrm{SD}$ or median with the IQR depending on the normal distribution of the numerical variables. Differences among three groups (SR, paroxysmal AF and persistent $\mathrm{AF}$ ) were evaluated using Fisher's exact test, $\chi^{2}$ test, one-way analysis of variance test with LSD corrections for multiple comparisons, or independent samples Kruskal-Wallis test depending on the normal distribution of the variables. Covariates used in the regression model included demographics, laboratory parameter, comorbidities and PE severity indexes. Statistically different variables were screened out and included in multivariate regressions. AF risk factors were analysed by binary logistic regression and carried out with the enter method. Kaplan-Meier analysis was used to compare the probability of 6-month survival and the log-rank test was used for comparison. Cox regression analysis was used to identify predictors of 6-month survival in patients discharged and carried out with the enter method. A value of $p<0.05$ was considered statistically significant.

\section{Patient and public involvement}

No patient was involved in this study.

\section{RESULTS}

\section{Clinical characteristics}

In the present real-world single-centre cohort, 590 aPE patients were included consecutively over a 2-year period, and $10.1 \%(71 / 702)$ of them suffered from $\mathrm{AF}$ on admission. The prevalence of new-onset $\mathrm{AF}$ in the present study was $7.7 \%$ (54/702). The incidence of paroxysmal AF and persistent $\mathrm{AF}$ are $3.3 \%$ (23/702) and $4.4 \%(31 / 702)$, respectively. Patients with $\mathrm{AF}$ at admission were older and with more comorbidities. In the study cohort, a statistically significant difference in age was observed between the paroxysmal AF group and the SR group. The average age of the paroxysmal AF group was 7 years older than that in the SR group ( $74 \pm 10$ vs $67 \pm 15, \mathrm{p}<0.05)$. The heart rate of the persistent AF group was faster $(96 \pm 30$ vs $85 \pm 14$, p $<0.01)$ than the SR group. No statistical difference in gender and arterial blood gas analysis was observed among the three groups. The SR group had a significantly lower NT-proBNP and cTNI-ultra than the persistent AF $(\mathrm{p}<0.05)$. However, the level of D-dimers was lower in the persistent AF group than in the SR group $(p<0.01)$. The proportion of pulmonary infection, ischaemic heart disease, congestive heart failure and chronic cor pulmonale in the AF group were significantly higher than that in the SR group $(\mathrm{p}<0.05)$, but the proportion of VTE was lower than that in the SR group $(p<0.05)$. In echocardiography parameters, the left ventricular ejection fraction (LVEF) in the SR group was higher than that in the AF group ( $52 \% \pm 7 \%$ vs $46 \pm 8 \%, \mathrm{p}<0.01$ ), but the left atrium diameter (LAD) and the left ventricular end-diastolic dimension were lower than those in 
Table 2 Treatment and outcome

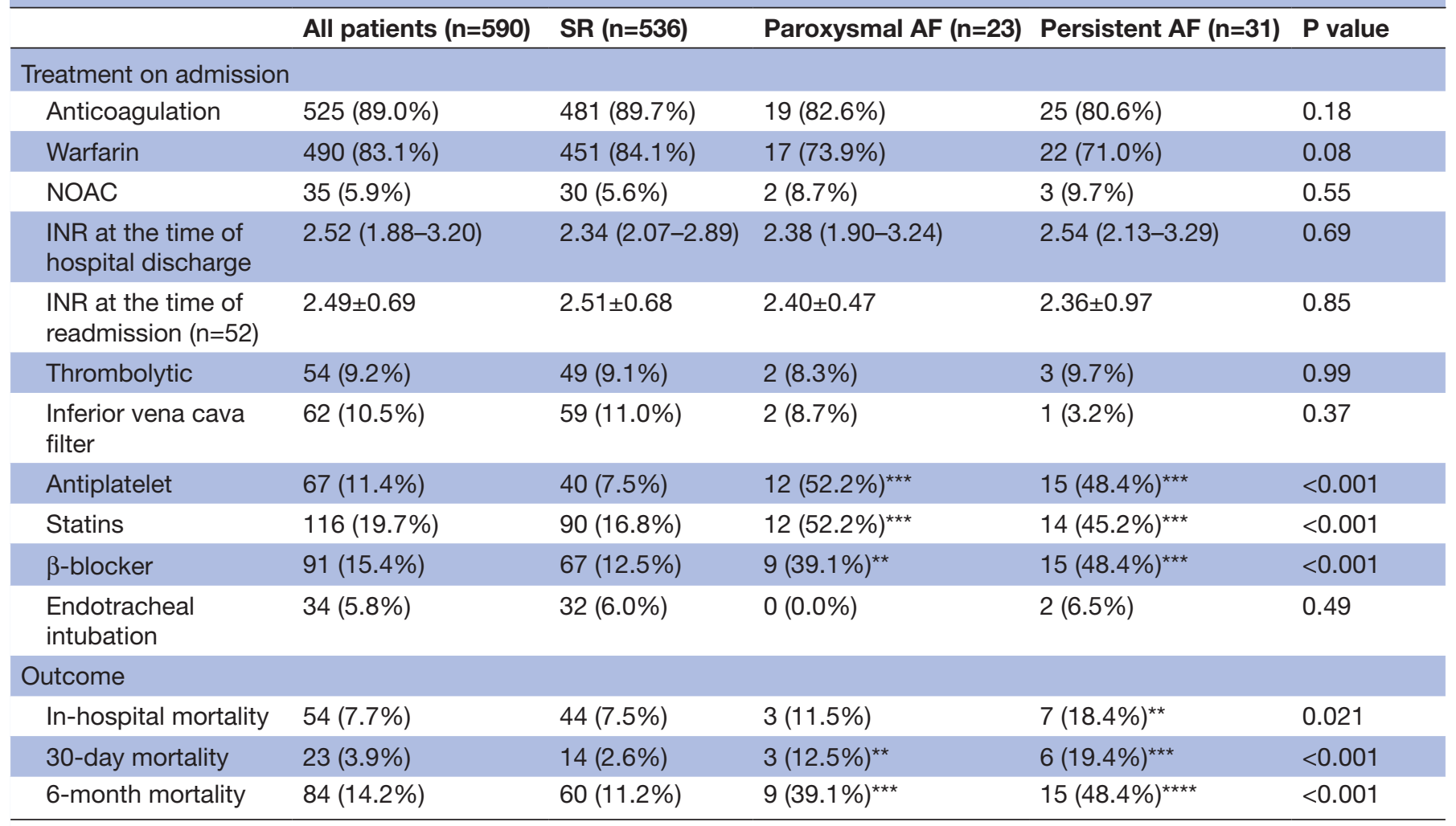

${ }^{*},{ }^{* *},{ }^{* \star *}$ : AF group vs SR group, $p<0.05, p<0.01$ and $p<0.001$, respectively.

$A F$, atrial fibrillation; INR, international normalised ratio; NOAC, novel oral anticoagulant; SR, sinus rhythm.

the persistent AF group $(\mathrm{p}<0.05)$. In addition, the right atrium diameter in the paroxysmal group was higher than that in the SR group $(\mathrm{p}<0.01)$. When it comes to risk stratification, although no significant difference in sPESI was found among the three groups, the incidence of massive $\mathrm{PE}$ in the persistent AF group was the highest $(\mathrm{p}<0.01)$ (table 1$)$. As regards the treatment, the AF group was subjected to $\beta$-blockers, statins and antiplatelet therapy in significantly higher proportions than the SR group $(\mathrm{p}<0.01)$ (table 2$)$.

\section{Subgroup analysis of the massive PE and risk factors for new- onset AF}

Considering that the AF group has a high incidence of massive $\mathrm{PE}$, we conducted a subgroup analysis. In the massive PE group, the LVEF in the paroxysmal AF group was significantly lower than that in the SR group $(p<0.05)$. The proportion of congestive heart failure in the persistent AF group was higher than that in the SR group, and heart rate and LAD also showed the same trend $(\mathrm{p}<0.05)$. In the paroxysmal and the persistent $\mathrm{AF}$ group, the heart rate of the persistent $\mathrm{AF}$ group was significantly higher than that of the paroxysmal AF group $(\mathrm{p}<0.05)$ (table 3$)$. In the entire cohort, the multivariable analysis showed that massive PE (OR 2.67; $95 \%$ CI 1.19 to $5.99 ; \mathrm{p}=0.017)$, ischaemic heart disease (OR 3.25; 95\% CI 1.65 to $6.39 ; \mathrm{p}<0.001)$ and congestive heart failure (OR 3.33; 95\% CI 1.81 to 6.12 ; p $<0.001$ ) were independent predictors of new-onset AF (table 4).

\section{Prognostic relevance of new-onset AF on admission}

During the follow-up, one patient died from other causes, and 12 patients were censored. The follow-up rate was $98 \%(578 / 590)$. The survival analysis was performed on 578 patients with complete data. The overall 30-day survival rate was $96.1 \%(567 / 590)$, and the 6 -month survival rate was $85.8 \%(506 / 590)$. The 30-day survival rates in the SR group, paroxysmal AF group and persistent AF group were 97.4\% (522/536), $87.5 \%(20 / 23)$ and $80.6 \%(25 / 31)$, respectively. The 6 -month survival rates in the SR group, paroxysmal AF group and persistent AF group were 88.8\% (476/536), $60.9 \%(14 / 23)$ and $51.6 \%(16 / 31)$, respectively (table 2). Kaplan-Meier analysis showed that the survival rate of the paroxysmal and persistent $\mathrm{AF}$ groups was significantly lower than the SR group within 6 months (60.9\% and $51.6 \%$ vs $88.8 \%, \mathrm{p}<0.001$ ) (figure 2). Cox survival analysis revealed that new-onset persistent $\mathrm{AF}$ (OR 2.36; 95\% CI 1.22 to 4.55; $\mathrm{p}=0.011$ ), malignancy (OR 3.84; 95\% CI 2.13 to 6.95 ; $\mathrm{p}<0.001$ ), congestive heart failure (OR 2.77; 95\% CI 1.64 to 4.68 ; $\mathrm{p}<0.001$ ), ischaemic heart disease (OR 2.40; 95\% CI 1.43 to 3.99; $\mathrm{p}=0.001$ ), cerebral infarction (OR 1.93; 95\% CI 1.09 to $3.40 ; \mathrm{p}=0.023$ ) and massive PE (OR 2.76; $95 \%$ CI 1.51 to 
Table 3 Baseline demographic and laboratory parameters with and without new-onset AF in massive PE (only showed statistically different indicators)

\begin{tabular}{llllll}
\hline & All patients $(\mathbf{n}=\mathbf{1 2 5})$ & SR $(\mathbf{n}=\mathbf{1 0 1})$ & Paroxysmal AF $(\mathbf{n}=\mathbf{1 1})$ & Persistent AF $(\mathbf{n}=\mathbf{1 3})$ & P value \\
\hline Congestive heart failure & $41(36.6 \%)$ & $26(29.2 \%)$ & $5(50.0 \%)$ & $10(76.9 \%)^{* *}$ & 0.003 \\
Diabetes & $7(6.5 \%)$ & $3(3.4 \%)$ & $2(20.0 \%)$ & $2(15.4 \%)$ & 0.042 \\
Heart rate (bpm) & $87 \pm 20$ & $85 \pm 16$ & $88 \pm 10$ & $105 \pm 37^{* *} \#$ & 0.004 \\
LVEF (\%) & $49(45-54)$ & $49(46-54)$ & $45(39-50)^{\star}$ & $46(42-49)$ & 0.009 \\
Chronic cor pulmonale & $5(4.5 \%)$ & $2(2.2 \%)$ & $1(10.0 \%)$ & $2(15.4 \%)$ & 0.068 \\
LAD & $38(30-45)$ & $36(30-44)$ & $39(36-48)$ & $47(39-54)^{\star}$ & 0.019 \\
History of VTE & $33(29.5 \%)$ & $31(34.8 \%)$ & $1(10.0 \%)$ & $1(7.7 \%)$ & 0.049 \\
\hline
\end{tabular}

${ }^{*},{ }^{* *},{ }^{* * *}$ : AF group vs SR group, $p<0.05, p<0.01$ and $p<0.001$, respectively.; \#: paroxysmal AF group vs persistent $A F$ group, $p<0.05$. $A F$, atrial fibrillation; LAD, left atrium diameter; LVEF, left ventricular ejection fraction; PE, pulmonary embolism; SR, sinus rhythm; VTE, venous thromboembolism.

$5.03 ; \mathrm{p}=0.001)$ were independent predictors of mortality within 6 month (table 5 ).

\section{DISCUSSION}

This present real-world single-centre cohort study identified several risk factors for new-onset $\mathrm{AF}$ in patients with aPE. In particular, massive $\mathrm{PE}$, ischaemic heart disease and congestive heart failure were associated with higher odds of new-onset AF. In addition, newonset persistent $\mathrm{AF}$ was an independent predictor for 6-month all-cause mortality in PE patients.

$\mathrm{PE}$ is a different clinical manifestation of VTE. Previous studies found that only $50 \%-70 \%$ of PE patients have VTE, but the proportion of AF in PE patients without a history of VTE reaches $54 \% .^{13}$ According to the largest population-based autopsy cohort, the thrombosis of the right atrium is difficult to assess. However, it is as common as the thrombosis of the left atrium. Of note, $38 \%$ of patients with intracardiac thrombosis had a manifest PE, and more than half had right-side intracardiac thrombosis. ${ }^{14}$ In addition, several studies suggested that isolated PE may arise from right atrial thrombi due to AF. ${ }^{15} 16$ Intriguingly, our study demonstrated that the prevalence of VTE was different in SR and AF groups. In those patients whose embolus origin cannot be determined, we speculated that it may be associated with those 'silence AF' that were not previously diagnosed.

Table 4 Predictors of new-onset AF after acute PE in univariate and multivariate model

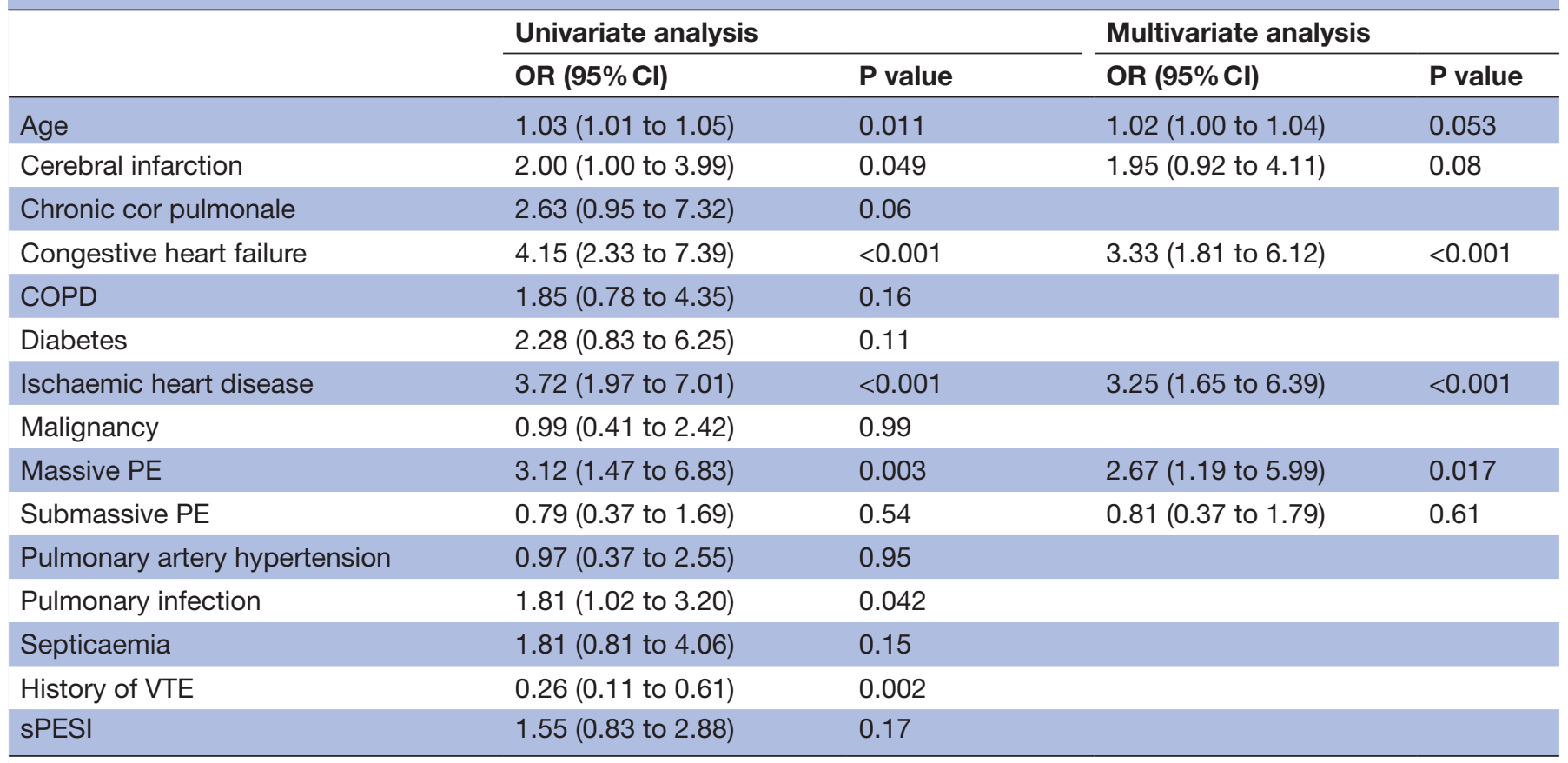

AF, atrial fibrillation; COPD, chronic obstructive pulmonary disease; PE, pulmonary embolism; sPESI, Simplified Pulmonary Embolism Severity Index; VTE, venous thromboembolism. 


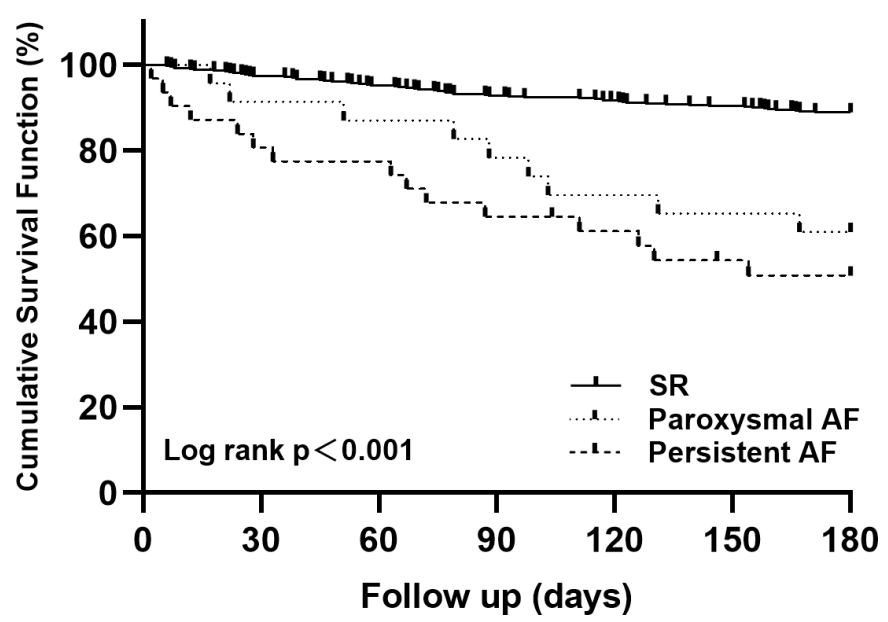

Figure 2 Kaplan-Meier curve depicting all-cause mortality for paroxysmal AF and persistent AF. AF, atrial fibrillation; SR, sinus rhythm. No statistical difference was found between paroxysmal AF and persistent AF. Paroxysmal AF (HR 3.75, $95 \% \mathrm{Cl} 1.01$ to 13.90$)$ and persistent $\mathrm{AF}$ (HR 5.74, 95\% Cl 1.76 to 18.72 ) have significantly higher mortality than sinus rhythm.

New-onset AF may be triggered by acute, potentially reversible precipitants. It has been confirmed that right ventricular failure and stretch injuries may trigger $\mathrm{AF}^{1718}$ Nevertheless, limited data are available on the risk factor of new-onset $\mathrm{AF}$ in PE patients. It has been reported age, congestive heart failure as independent predictors for subsequent AF in PE cohort. ${ }^{19-21}$ Massive PE, which causes a sharp increase in afterload and stretch injuries, could also be associated with AF. Our study confirmed that massive PE was a risk factor for new-onset AF. In addition, we also found that patients with congestive heart failure or ischaemic heart disease had a higher prevalence of $\mathrm{AF}$ compared with patients without that, which is consistent with previous studies.

To find out who is susceptible to paroxysmal or persistent $\mathrm{AF}$ in massive $\mathrm{PE}$ group, we reclassified patients using risk stratification proposed by AHA/ACC and conducted an analysis of subgroups. In massive PE group, we found that when patients have congestive heart failure, fast heart rate and $\mathrm{LAD}$, they were susceptible to persistent $\mathrm{AF}$. We surmised that the occurrence of persistent AF may be related to the severity of congestive heart failure. When cardiac function or afterload cannot be improved, the occurrence of myocardial remodelling provides a substrate for the development of persistent AF. Considering that we included small samples, more in-depth research may be needed.

Our cohort demonstrated that in-hospital and 6-month mortality were higher than the previous studies. ${ }^{19-22} \mathrm{Ng}$ et al found that PE with baseline AF had higher mortality than subsequent $\mathrm{AF}$ in the first year $(23.7 \%$ vs $9.5 \%)$ and the fifth year $(45.4 \%$ vs $34.9 \%)$, but the latter had

Table 5 Univariate and enter multivariate Cox hazard analysis for the predictors of 6-month mortality

\begin{tabular}{|c|c|c|c|c|}
\hline & \multicolumn{2}{|l|}{ Univariate analysis } & \multicolumn{2}{|c|}{ Multivariate analysis } \\
\hline & OR $(95 \% \mathrm{Cl})$ & $P$ value & OR (95\% Cl) & $P$ value \\
\hline Age & 1.01 (0.99 to 1.03$)$ & 0.18 & 1.01 (0.99 to 1.03 ) & 0.37 \\
\hline NT-proBNP & 2.44 (1.37 to 4.35$)$ & 0.002 & 0.88 (0.45 to 1.72$)$ & 0.71 \\
\hline cTNI-ultra & 0.88 (0.53 to 1.49$)$ & 0.64 & & \\
\hline LVEF & 0.99 (0.95 to 1.02$)$ & 0.35 & & \\
\hline Paroxysmal AF & $4.04(2.00$ to 8.14$)$ & $<0.001$ & 1.43 (0.65 to 3.18$)$ & 0.37 \\
\hline Persistent AF & 5.93 (3.36 to 10.46$)$ & $<0.001$ & 2.36 (1.22 to 4.55$)$ & 0.011 \\
\hline Ischaemic heart disease & 3.02 (1.88 to 4.86$)$ & $<0.001$ & 2.40 (1.43 to 3.99$)$ & 0.001 \\
\hline Malignancy & 2.82 (1.71 to 4.67$)$ & $<0.001$ & 2.77 (1.57 to 4.87$)$ & $<0.001$ \\
\hline Septicaemia & 1.45 (0.75 to 2.81$)$ & 0.27 & & \\
\hline COPD & 1.48 (0.74 to 2.95$)$ & 0.27 & & \\
\hline Pulmonary artery hypertension & $1.50(0.80$ to 2.84$)$ & 0.21 & & \\
\hline History of VTE & $0.70(0.42$ to 1.15$)$ & 0.16 & & \\
\hline Cerebral infarction & 2.22 (1.34 to 3.67$)$ & 0.002 & 1.93 (1.09 to 3.40$)$ & 0.023 \\
\hline Diabetes & $1.38(0.56$ to 3.41$)$ & 0.49 & & \\
\hline Pulmonary infection & 1.36 (0.87 to 2.12$)$ & 0.18 & & \\
\hline Chronic cor pulmonale & 1.90 (0.83 to 4.35$)$ & 0.13 & & \\
\hline Congestive heart failure & 4.06 (2.64 to 6.24$)$ & $<0.001$ & 2.50 (1.51 to 4.12$)$ & $<0.001$ \\
\hline Massive PE & 3.50 (1.98 to 6.19$)$ & $<0.001$ & 2.76 (1.51 to 5.03 ) & 0.001 \\
\hline Submassive PE & 0.69 (0.38 to 1.27$)$ & 0.24 & 0.68 (0.36 to 1.29$)$ & 0.24 \\
\hline
\end{tabular}

cTnl-ultra; cardiac troponin I ultra; AF, atrial fibrillation; COPD, chronic obstructive pulmonary disease; LVEF, left ventricular ejection fraction; NT-proBNP, amino-terminal pro-brain natriuretic peptide; PE, pulmonary embolism; VTE, venous thromboembolism. 
higher all-cause mortality during long-term follow-up. ${ }^{19}$ These results suggested that the subsequent AF may have an adverse effect on the long-term prognosis. Ebner et al reported that $\mathrm{AF}$ on admission with $\mathrm{PE}$ 1-year mortality was $17.5 \%$, and it do not affect the prognostic performance. ${ }^{20}$ However, they neither distinguished whether AF was subsequent or existed before, nor it was persistent or paroxysmal. In addition, $\mathrm{Ng}$ et al showed that subsequent AF with PE 6-month mortality was $10 \%,{ }^{19}$ while they did not investigate the effects of different $\mathrm{AF}$ types on the mortality. In our cohort, we found that the 6-month mortality of persistent AF group reached a staggering $48.4 \%$. These results implicit that there may be regional differences in the management of $\mathrm{AF}$ in $\mathrm{PE}$, and $\mathrm{AF}$ affects the prognosis of $\mathrm{PE}$ patients in multiple dimensions.

There is no consensus on the impact of AF on PE risk stratification. A recent study showed that the inclusion of AF in sPESI could improve the prediction of in-hospital adverse events. ${ }^{23}$ In another PE cohort, they found AF group had a high proportion of massive PE and in-hospital mortality. ${ }^{24}$ Indeed, the in-hospital mortality of PE combined with $\mathrm{AF}$ is $20 \%-32 \%$, and nearly half of the patients suffer from rightsided intracardiac thrombus. ${ }^{21}{ }^{25}$ The incidence of complications is also significantly higher in these patients than in the ones without $\mathrm{AF} .^{20} \mathrm{~A}$ meta-analysis performed on 10 studies, including 3007 patients with aPE, demonstrated that $\mathrm{AF}$ is associated with an increased risk of circulatory shock and death. ${ }^{26}$ Our research found that massive PE is a risk factor for AF. Therefore, the occurrence of AF may reflect the severity of PE to a certain extent. On the pathophysiology, providing the corresponding preload to the left heart by the right heart is essential to maintain haemodynamic stability and long-term survival in PE patients. However, the episode of AF aggravates haemodynamic disturbances. Furthermore, persistent fast ventricular rhythm causes progressive heart failure, which in turn causes death. In addition, persistent $\mathrm{AF}$, which provokes a hypercoagulable state and induces platelet aggregation, may aggravate the embolic area of PE. Eventually, the complex interaction between AF and PE leads to increased mortality.

Our result implied that the complementary treatment for comorbidities, especially the control of cardiovascular disease, could also be essential for the prognosis. In our cohort, the proportion of congestive heart failure $(48.1 \%$ vs $20.6 \%$ ) and ischaemic heart disease (31.5\% vs $26.5 \%)$ in the new-onset AF group was higher than that in the study of the Ebner et al. However, the use of $\beta$-blocker and antiplatelet ratios were lower than the previous studies when combined with cardiovascular disease. ${ }^{20}$ When patients had anaemia or chronic obstructive pulmonary disease, whether those relative contraindications affecting the use of $\beta$-blocker and antiplatelet is worthy of our thinking. In addition, some patients might be first diagnosed with cardiovascular disease in our cohort. These fatal complications or comorbidities affected the prognosis because patients were not appropriately examined or treated by the cardiologist. As regard patients with malignancy, they underwent more palliative treatment.
Due to economic factors, nearly $80 \%$ of patients used warfarin in our cohort. The ratios of INR values between 2.0 and 3.0 in the SR group, paroxysmal AF group and persistent AF group were $59.0 \%, 39.1 \%$ and $54.8 \%$, respectively. Therefore, the overall compliance rate of warfarin was unsatisfactory. Interestingly, among the $8.8 \%(52 / 590)$ of patients who were readmitted to our hospital, 26.9\% (14/52) had malignancy. The compliance rates of the SR group, paroxysmal AF group and persistent AF group were 42.9\% (18/42), 50.0\% (2/4) and $33.3 \%(2 / 6)$, respectively. This result suggested that their compliance rate was further reduced. Their all-cause mortality was $17.3 \%(9 / 52)$ within 6 months and $77.8 \%(7 / 9)$ of death did not meet the target INR. Since warfarin has a high risk of bleeding, patients with comorbidities have a high incidence of interrupting or adjusting the warfarin dose by themselves. A treatment range time $>70 \%$ could hardly be achieved..$^{27}$ In brief, interdisciplinary cooperation, outpatient management and follow-up are vital.

As regards the treatments, the following points should be underlined. Compared with warfarin, patients with $\mathrm{AF}$ treated with apixaban and dabigatran showed the low risk of subsequent VTE. ${ }^{28}{ }^{29}$ NOAC could be an alternative drug for PE. ${ }^{30}{ }^{31}$ The primary and secondary prevention of cardiovascular diseases should be enhanced after the diagnosis of aPE due to the high incidence of cardiovascular diseases. Besides, drugs used to treat cardiovascular diseases are also effective for PE, and the transition from acute treatment to long-term treatment after discharge may reduce mortality.

Our research has some strength. Previous studies did not distinguish AF types, ${ }^{20}$ while AF was more carefully divided into paroxysmal and persistent $\mathrm{AF}$ in this study. In addition, our study is currently the largest PE cohort study in China and provides sufficient detailed follow-up data. Besides, we first confirmed that new-onset persistent $\mathrm{AF}$ is an independent predictor affecting the prognosis of patients with PE. Our research also has some limitations. First, the study was based on the retrospective analysis of patient medical records. So, some data were missing, and the follow-up time was relatively short. Second, we could only get patients' all-cause mortality. The incidence of sudden death or cardiovascular mortality in the PE patients is unclear. Third, our data maybe not applicable for different cohorts in other developed countries. However, our results suggested that the PE patients with persistent $\mathrm{AF}$ should be paid more attention to improve the prognosis.

\section{CONCLUSION}

Massive PE, ischaemic heart disease and congestive heart failure are high-risk factors which were related to newonset $\mathrm{AF}$ in aPE. New-onset persistent $\mathrm{AF}$ was an independent predictor for 6-month all-cause mortality in PE patients. 
Contributors DL, SS and BY contributed to the conception of the study. $X L$ and CQ contributed to the design of the study. TY, LW and DL collected the raw data. DL wrote the first draft and was involved in the editing of the manuscript, which was critically reviewed by BY, QZ, TY and LW and QZ approved the final manuscript.

Funding The authors have not declared a specific grant for this research from any funding agency in the public, commercial or not-for-profit sectors.

Competing interests None declared.

Patient and public involvement Patients and/or the public were not involved in the design, or conduct, or reporting or dissemination plans of this research.

Patient consent for publication Consent obtained from parent(s)/guardian(s)

Ethics approval Because the patient's privacy was not violated in the study, the Ethics Committee agreed exemption applications of informed consent.

Provenance and peer review Not commissioned; externally peer reviewed.

Data availability statement All data relevant to the study are included in the article or uploaded as supplementary information. Raw data are not available for data sharing.

Open access This is an open access article distributed in accordance with the Creative Commons Attribution Non Commercial (CC BY-NC 4.0) license, which permits others to distribute, remix, adapt, build upon this work non-commercially, and license their derivative works on different terms, provided the original work is properly cited, appropriate credit is given, any changes made indicated, and the use is non-commercial. See: http://creativecommons.org/licenses/by-nc/4.0/.

ORCID iD

Dishiwen Liu http://orcid.org/0000-0001-5269-5754

\section{REFERENCES}

1 Keller K, Hobohm L, Ebner M, et al. Trends in thrombolytic treatment and outcomes of acute pulmonary embolism in Germany. Eur Heart $J$ 2019;118.

2 Wendelboe AM, Raskob GE. Global burden of thrombosis: epidemiologic aspects. Circ Res 2016;118:1340-7.

3 Cohen AT, Agnelli G, Anderson FA, et al. Venous thromboembolism (VTe) in Europe. the number of VTe events and associated morbidity and mortality. Thromb Haemost 2007;98:756-64.

4 Cohen AT, Tapson VF, Bergmann J-F, et al. Venous thromboembolism risk and prophylaxis in the acute hospital care setting (ENDORSE study): a multinational cross-sectional study. Lancet 2008;371:387-94.

5 Alikhan R, Peters F, Wilmott R, et al. Fatal pulmonary embolism in hospitalised patients: a necropsy review. J Clin Pathol 2004;57:1254-7.

6 Jiménez D, de Miguel-Díez J, Guijarro R, et al. Trends in the management and outcomes of acute pulmonary embolism: analysis from the RIETE registry. J Am Coll Cardiol 2016;67:162-70.

7 Lozano R, Naghavi M, Foreman K, et al. Global and regional mortality from 235 causes of death for 20 age groups in 1990 and 2010: a systematic analysis for the global burden of disease study 2010. Lancet 2012;380:2095-128.

8 Spencer FA, Goldberg RJ, Lessard D, et al. Factors associated with adverse outcomes in outpatients presenting with pulmonary embolism: the worcester venous thromboembolism study. Circ Cardiovasc Qual Outcomes 2010;3:390-4.

9 Slobodan O, Boris D, Bojana S, et al. Predictive value of heart failure with reduced versus preserved ejection fraction for outcome in pulmonary embolism. ESC Heart Fail 202010.1002/ehf2.13015. [Epub ahead of print: 16 Sep 2020].

10 Klok FA, Mos ICM, Broek L, et al. Risk of arterial cardiovascular events in patients after pulmonary embolism. Blood 2009;114:1484-8.
11 Konstantinides SV. 2014 ESC guidelines on the diagnosis and management of acute pulmonary embolism. Eur Heart $J$ 2014;35:997-1053.

12 Grupa Robocza Europejskiego Towarzystwa Kardiologicznego (ESC) do spraw rozpoznawania i postepowania w ostrej zatorowości płucnej, Konstantinides S, Torbicki A, et al. [2014 ESC Guidelines on the diagnosis and management of acute pulmonary embolism]. Kardiol Pol 2014;72:997-1053.

13 Keller K, Prochaska JH, Coldewey M, et al. History of deep vein thrombosis is a discriminator for concomitant atrial fibrillation in pulmonary embolism. Thromb Res 2015;136:899-906.

14 Ogren $\mathrm{M}$, Bergqvist $\mathrm{D}$, Eriksson $\mathrm{H}$, et al. Prevalence and risk of pulmonary embolism in patients with intracardiac thrombosis: a population-based study of 23796 consecutive autopsies. Eur Heart J 2005:26:1108-14.

15 Enga KF, Rye-Holmboe I, Hald EM, et al. Atrial fibrillation and future risk of venous thromboembolism:the Tromsø study. J Thromb Haemost 2015;13:10-16.

16 Morella P, Sacco M, Carafa M, et al. Permanent atrial fibrillation and pulmonary embolism in elderly patients without deep vein thrombosis: is there a relationship? Aging Clin Exp Res 2019;31:1121-8.

17 Geibel A, Zehender M, Kasper W, et al. Prognostic value of the ECG on admission in patients with acute major pulmonary embolism. Eur Respir J 2005;25:843-8.

18 Aziz EF, Kukin M, Javed F, et al. Right ventricular dysfunction is a strong predictor of developing atrial fibrillation in acutely decompensated heart failure patients, ACAP-HF data analysis. $J$ Card Fail 2010;16:827-34.

$19 \mathrm{Ng}$ ACC, Adikari D, Yuan D, et al. The prevalence and incidence of atrial fibrillation in patients with acute pulmonary embolism. PLOS One 2016;11:e0150448.

20 Ebner M, Rogge NIJ, Parwani AS, et al. Atrial fibrillation is frequent but does not affect risk stratification in pulmonary embolism. $J$ Intern Med 2020;287:100-13.

21 Krajewska A, Ptaszynska-Kopczynska K, Kiluk I, et al. Paroxysmal atrial fibrillation in the course of acute pulmonary embolism: clinical significance and impact on prognosis. Biomed Res Int 2017;2017:5049802

22 Ng ACC, Chung T, Yong ASC, et al. Long-term cardiovascular and noncardiovascular mortality of 1023 patients with confirmed acute pulmonary embolism. Circ Cardiovasc Qual Outcomes 2011;4:122-8.

23 Tang R-B, Jing Y-Y, Xu Z-Y, et al. New-Onset atrial fibrillation and adverse in-hospital outcome in patients with acute pulmonary embolism. Semin Thromb Hemost 2020;46:887-94.

24 Yang Y, Liu B, Taylor J, et al. Prevalence and clinical impact of atrial fibrillation in patients with pulmonary embolism. South Med $J$ 2020;113:93-7.

25 Kukla P, Mclntyre WF, Koracevic G, et al. Relation of atrial fibrillation and right-sided cardiac thrombus to outcomes in patients with acute pulmonary embolism. Am J Cardiol 2015;115:825-30.

26 Shopp JD, Stewart LK, Emmett TW, et al. Findings from 12-lead electrocardiography that predict circulatory shock from pulmonary embolism: systematic review and meta-analysis. Acad Emerg Med 2015;22:1127-37.

27 De Caterina R, Husted S, Wallentin L, et al. General mechanisms of coagulation and targets of anticoagulants (Section I). Position Paper of the ESC Working Group on Thrombosis--Task Force on Anticoagulants in Heart Disease. Thromb Haemost 2013;109:569-79.

28 Lutsey PL, Norby FL, Zakai NA, et al. Oral anticoagulation therapy and subsequent risk of venous thromboembolism in atrial fibrillation patients. Curr Med Res Opin 2019;35:837-45.

29 Bassand J-P, Virdone S, Goldhaber SZ, et al. Early risks of death, Stroke/Systemic embolism, and major bleeding in patients with newly diagnosed atrial fibrillation. Circulation 2019;139:787-98.

30 Becattini C, Agnelli G. Treatment of venous thromboembolism with new anticoagulant agents. J Am Coll Cardiol 2016;67:1941-55.

31 van Es N, Coppens M, Schulman S, et al. Direct oral anticoagulants compared with vitamin $\mathrm{K}$ antagonists for acute venous thromboembolism: evidence from phase 3 trials. Blood 2014; 124:1968-75. 